\title{
Absence of chromosome 17 polysomy in breast cancer: analysis by CEP17 chromogenic in situ hybridization and multiplex ligation-dependent probe amplification
}

\author{
Cathy B. Moelans · Roel A. de Weger • \\ Paul J. van Diest
}

Received: 17 July 2009/Accepted: 29 August 2009/Published online: 18 September 2009

(C) Springer Science+Business Media, LLC. 2009

\begin{abstract}
Amplification of the HER2 gene, present in $15-30 \%$ of breast carcinomas, correlates with poor outcome and is an indication for treatment with trastuzumab. Standard testing methods for HER2 amplification are fluorescence (FISH) or chromogenic in situ hybridization (CISH). In FISH/CISH scoring, correction for chromosome 17 polysomy is believed to be critical for determination of true HER2 amplification as opposed to increased chromosome 17 copy number. The term "polysomy 17" is widely used and defined as $\geq 3$ copies of the chromosome 17 centromere (probe CEP17, D17Z1). Thus, the centromere is assumed to be representative for the entire chromosome. This study aimed to investigate the frequency of polysomy 17 and its association with HER2 amplification in 111 invasive breast cancer patients by CEP17 CISH and by copy number analysis of a set of 17 genes along chromosome 17 using multiplex ligation-dependent probe amplification (MLPA).

Chromosome 17 usually showed a complex pattern of gains and losses by MLPA, unrelated to the copy number status of the centromere. Increase in centromere 17 copy number (denoted "polysomy 17"), as assessed by CEP17 $\mathrm{CISH}$, was found in $19 \%$ of the patients. Of these patients, $60 \%$ also showed amplification of HER 2 measured by MLPA. However, none of the 111 patients showed a true polysomy of chromosome 17 by MLPA. Only two patients
\end{abstract}

Electronic supplementary material The online version of this article (doi:10.1007/s10549-009-0539-2) contains supplementary material, which is available to authorized users.

C. B. Moelans $(\bowtie) \cdot$ R. A. de Weger · P. J. van Diest Department of Pathology, University Medical Centre Utrecht, Heidelberglaan 100, PO Box 85500, 3508 GA Utrecht, The Netherlands

e-mail: cmoelans@umcutrecht.nl
(1.8\%) had a possible gain of 17q. Amplification of $17 \mathrm{p}$ was not found in any of the patients, although a possible loss of $17 p$ was found in one patient. In conclusion, this extensive analysis of amplicons along chromosome 17 shows that true polysomy of chromosome 17, either of the whole chromosome or of the short or the long arm, is very rare in invasive breast cancer. Abnormal CEP17 copy numbers may therefore actually stem from high level gains or amplification of CEP17 regardless of copy number gains of the short and long arms of chromosome 17 and, at least in some cases, correction with CEP17 probes may provide misleading HER2 gene status assessment results.

Keywords Breast cancer - MLPA - CEP17 - Polysomy · HER2

\section{Introduction}

Several genes have been shown to be implicated in the development, progression and response to therapy of invasive breast cancer. Among these, HER-2/neu is likely the most important proto-oncogene. HER2 is located on chromosome 17q21 and encodes an epidermal growth factor receptor family protein involved in signal transduction [1, 2]. Amplification of the HER2 gene, which is present in about $15-30 \%$ of breast carcinomas and leads to protein overexpression, correlates with a poor outcome [3] and is an indication for treatment with the recombinant humanized monoclonal anti-HER2 antibody trastuzumab $[4,5]$. Standard testing methods include, besides immunohistochemistry, analysis of HER2 gene copy number by fluorescence (FISH) or chromogenic in situ hybridization (CISH). In FISH (and to a lesser extent CISH) scoring, correction for chromosome 17 polysomy is believed to be 
critical for determination of true HER2 gene amplification as opposed to increased chromosome 17 copy number [6, 7]. The term "polysomy 17 " is widely used and is defined as $\geq 3$ copies of the chromosome 17 centromere (probe CEP17, D17Z1). Thus, the centromere is assumed to be representative for the entire chromosome. Reported incidence of polysomy 17 ranges from 10 to $49 \%$, depending on the criteria used to define polysomy [8]. In a recent study by Bartlett et al. [9], it was stated that the presence of polysomy 17 , as established by CEP17 FISH rather than HER2 and TOP2A amplification, was predictive for response to anthracyclins. This further underlines the importance of assessing chromosome 17 copy number increase. Overall, chromosome 17 is one of the smallest and the second most densely gene-loaded human chromosome. It is rearranged in at least $30 \%$ of breast cancers with short and long arms differing in the type of events they harbor [10, 11]. Chromosome $17 \mathrm{p}$ is mainly involved in losses, some of them possibly focal, whereas comparative genomic hybridization $(\mathrm{CGH})$ on $17 \mathrm{q}$ shows complex combinations of overlapping gains and losses. Therefore, increase in the centromeric region of chromosome 17 as detected by FISH/CISH may not at all reflect "polysomy" 17 and thereby be unsuitable to correct for $17 \mathrm{q}$ status. Also, the long arm of chromosome $17(17 q)$ is frequently characterized by a "firestorm" pattern in CGH studies (many narrow peaks of amplification and/or loss), but 5-10\% of the firestorms do not include amplification of HER2 giving weight to the notion that other loci in the region may contribute to oncogenesis [12]. There have been many $\mathrm{CGH}$ studies to characterize breast cancer in terms of gene and class discovery [12-16], and although array CGH will undoubtedly have a number of clinical applications in the future, it is still too early to be used routinely by clinicians because it is still a costly and labor intensive technique that requires a relatively large amount of sample DNA and trained personnel to deal with the complexity of the data.

In the present study, we used an easier and faster highthroughput technique, called multiplex ligation-dependent probe amplification (MLPA), to characterize chromosome 17 status in paraffin-embedded invasive breast cancer samples. We specifically investigated the frequency of true polysomy 17 and the presence of co-amplifications. MLPA can simultaneously determine copy number gains and/or losses of multiple genes [17] (the centromere region included) along chromosome 17 . In previous studies, we obtained promising results with MLPA in comparison with ISH [18] and evaluated this technique to simultaneously determine copy number changes of HER2 and TOP2A, a gene that has shown involvement in the response to anthracyclins by some groups $[19,20]$. To analyze the centromere status of chromosome 17, we performed CEP17 chromogenic in situ hybridization. Additionally, we evaluated WSB1, located very near the centromere region at $17 q 11.1(22,645,233-22,664,772 \mathrm{bp}$ from pter), as an alternative for CEP17 in our MLPA analysis.

\section{Methods}

Patient material

Tissue samples of 111 invasive breast cancer patients were randomly selected from a previous study at the Department of Pathology of the University Medical Center in Utrecht [18]. Anonymous use of redundant tissue for research purposes is part of the standard treatment agreement with patients in our hospital [21]. All tissue samples were analyzed with immunohistochemistry (IHC) to assess HER2 protein expression and MLPA to determine gene copy number alternations. Subsequently, CEP17 analysis by CISH was performed on 106 samples; the other 5 samples were excluded from analysis as there was too little material left.

\section{Immunohistochemistry}

IHC for HER2 was performed using the Hercept test (Dako, Glostrup, Denmark) according to the manufacturers' instructions on $4-\mu$ m-thick sections from neutral buffered formaldehyde fixed tissue blocks. IHC membrane staining was semiquantitatively scored as negative (0), weakly positive $(1+)$, positive $(2+)$ and strongly positive $(3+)$ according to the DAKO FDA-approved scoring system. Areas with intraductal carcinoma were excluded from the evaluation and cytoplasmic staining was ignored. Interpretation of staining was done by two experienced breast pathologists. As control, a small tissue array containing a $0,1+, 2+$ and $3+$ breast tumor samples was taken along on the same slide as the tumor to be analyzed. Appropriate negative controls were used throughout.

Multiplex ligation-dependent probe amplification

Invasive tumor areas as identified on serial $H \& E$ sections were harvested from two to four whole 4- $\mu$ m-thick paraffin sections (corresponding to $\sim 1 \mathrm{~cm}^{2}$ tumor tissue) with a scalpel. DNA was isolated from these tissue fragments by $1 \mathrm{~h}$ incubation in proteinase $\mathrm{K}(10 \mathrm{mg} / \mathrm{ml}$; Roche, Almere, The Netherlands) at $56^{\circ} \mathrm{C}$ followed by boiling for $10 \mathrm{~min}$. This DNA solution (50-100 $\mu \mathrm{l})$ was, after centrifugation, used in the MLPA analysis according the manufacturers' instructions, using the P004-B1 kit (MRC Holland, Amsterdam, The Netherlands). The contents of this kit are depicted in Table 1. It contains probes for 17 chromosome 
Table 1 Genes on chromosome 17 targeted by probes in the P004B1 MLPA kit (MRC Holland)

\begin{tabular}{llll}
\hline Gene & Location & $\begin{array}{l}\text { Distance } \\
\text { to pter }\end{array}$ & $\begin{array}{l}\text { Number } \\
\text { of probes }\end{array}$ \\
\hline PAFAH1B1 & $17 \mathrm{p} 13.3$ & 2.530 & 1 \\
PMP22 & $17 \mathrm{p} 12$ & 15.083 & 1 \\
TOM1L2 & $17 \mathrm{p} 11.2$ & 17.727 & 1 \\
WSB1 & $17 \mathrm{q} 11.1$ & $22.654 / 663 / 663$ & 3 \\
NOS2A & $17 \mathrm{q} 11.1$ & 23.133 & 1 \\
TRAF4 & $17 \mathrm{q} 11.2$ & 24.098 & 1 \\
CPD & $17 \mathrm{q} 11.2$ & 25.795 & 1 \\
RNF135 & $17 \mathrm{q} 11.2$ & 26.336 & 1 \\
PEX12 & $17 \mathrm{q} 21$ & 30.928 & 1 \\
NEUROD2 & $17 \mathrm{q} 21$ & 35.014 & 1 \\
ERBB2/HER2 & $17 \mathrm{q} 21$ & $35.118 / 133 / 137$ & 3 \\
RARA & $17 \mathrm{q} 21$ & 35.762 & 1 \\
TOP2A & $17 \mathrm{q} 21$ & $35.817 / 818 / 823$ & 3 \\
BRCA1 & $17 \mathrm{q} 21.31$ & $38.469 / 496$ & 2 \\
SGCA & $17 \mathrm{q} 21.33$ & 45.603 & 1 \\
GH1 & $17 \mathrm{q} 24.1$ & 59.350 & 1 \\
METRNL & $17 \mathrm{q} 25$ & 78.636 & 1 \\
REFERENCE PROBES & & & 15 \\
\hline
\end{tabular}

17 genes: 3 on $17 \mathrm{p}$, one very close to the centromeric region (WSB1), 13 on $17 \mathrm{q}$, as well as 15 control probes. All tests were performed in duplicate in an ABI 9700 PCR machine. PCR products were analyzed on an ABI310 capillary sequencer (Applied Biosystems, Foster City, CA, USA). Gene copy numbers were analyzed using Genescan and Coffalyser (version 7.0) software. For genes with more than one probe present in the kit, the mean of all the probe peaks of this gene in duplicate was calculated. If this mean value was below 0.7 the respective gene was defined as lost, a value between 0.7 and 1.3 was defined as normal, $1.3-2.0$ as low level amplification and values $>2.0$ as high level amplified, according to the definitions in the Coffalyser software [22].

\section{Centromere 17 chromogenic in situ hybridization}

CEP17 CISH was performed using SpoT-Light chromosome 17 centromeric probe (Zymed, San Francisco, 840500) and SpoT-Light CISH centromere detection kit (Zymed, 84-9248) according to the manufacturer's instructions. CISH was mainly performed on $4-\mu \mathrm{m}$-thick tissue microarray sections $(n=57)$, supplemented with whole sections $(n=49)$ for further cases not present on the tissue microarray. Polysomy was defined as three or more copies of CEP17, counted in at least 30 tumor cells.
Statistics

Associations between two categorical variables were examined using chi-square with continuity correction and Fisher's exact tests if necessary. Unsupervised hierarchical cluster analysis (Euclidean distance, average linkage analysis) was performed using the open-source $\mathrm{R}$ software (version 2.9.1, http://www.r-project.org).

\section{Results}

Copy numbers for genes on chromosome 17 by MLPA

Supplementary Table 1 shows the copy numbers for the 17 genes along chromosome 17 for all cases. Most individual cases showed a complex pattern of amplifications and losses over chromosome 17. Frequencies of amplification and losses along chromosome 17 are depicted in Fig. 1. There were four major regions of amplification: 17q11.111.2 with the WSB1, NOS2A, TRAF4 and CPD genes; $17 \mathrm{q} 12$ with the PEX12, NEUROD2 and HER2 genes; 17q21.2 with TOP2A; and 17q21.33-q25.3 with SGCA, GH1 and METRNL. High level amplifications (ratio >2.0) are mainly localized in the second region containing HER2 (73\% of its amplifications were high level). We found two regions on chromosome 17 with frequent loss: 17p11.2-p12 containing TOM1L2 and PMP22 and 17q21.2 containing RARA.

\section{Centromere analysis}

CEP17 analysis in 106 patients showed copy number increase (ranging from three copies/nucleus to large clusters with $>10$ copies/nucleus) in 20/104 patients (19.2\%); two samples were inconclusive. Copy number analysis of the centromere region by MLPA (three WSB1 probes) showed increased copy number in $16 \%(18 / 111)$ of all patients; $33 \%$ of these $(6 / 18)$ showed a high level amplification. Overall, the concordance between CEP17 and WSB1 copy number status was $92.3 \%$ (96/104) (see Table 2).

Polysomy analysis

None of the 111 patients showed a true polysomy of whole chromosome 17, as reflected by a copy number increase of all probes along chromosome 17 . Polysomy of whole $17 \mathrm{p}$ as defined by amplification of all three probes on $17 \mathrm{p}$ was not found in any of the patients either, although possible loss of whole $17 \mathrm{p}$ was found in one patient that showed loss of all three $17 \mathrm{p}$ probes. This patient had a normal 
Fig. 1 Chromosome 17 copy number aberrations by MLPA in 111 breast cancer patients. Top percentage of patients showing amplifications (ratio $>1.3$, white) and losses (ratio $<0.7$, black) for all the chromosome 17 probes of the MLPA kit. Bottom percentage of patients showing amplifications $(>1.3$, white) and high level

amplifications (ratio $>2.0$,

black) for all the chromosome 17 probes of the MLPA kit

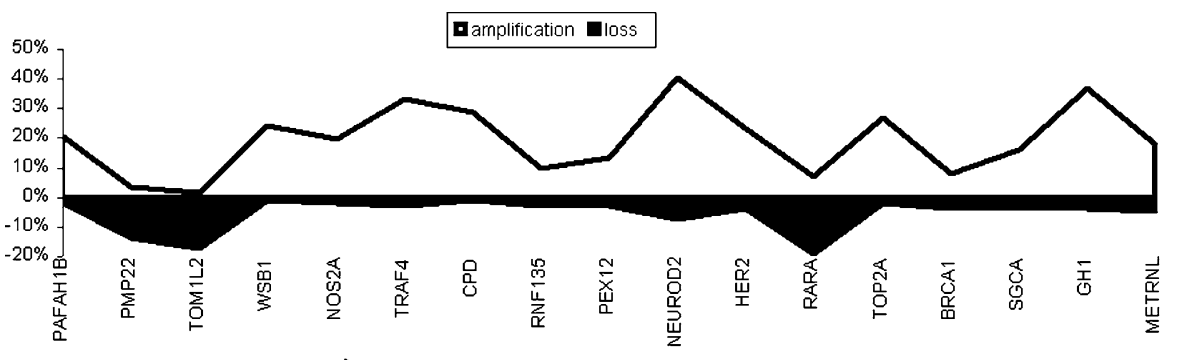

玄

\begin{tabular}{|c|c|c|c|c|c|c|c|c|c|c|c|}
\hline $17 p 13$ & $\mathrm{p} 12$ & p11.2 & $\vec{E} \bar{E}$ & $q 11.2$ & $q 12$ & & $q 21$ & $q 22$ & $q 23$ & $q 24$ & $17 q 25$ \\
\hline 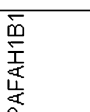 & $\underset{\mathbb{N}}{\mathbb{N}}$ & & 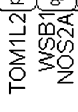 & 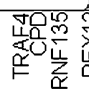 & 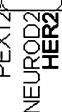 & 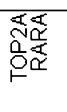 & $\begin{array}{l}\bar{Z} \\
\text { U } \\
\text { 舀 }\end{array}$ & & & $\frac{\bar{T}}{0}$ & $\begin{array}{l}\frac{\vec{Z}}{\underline{\underline{Y}}} \\
\frac{\mathrm{W}}{\Sigma}\end{array}$ \\
\hline
\end{tabular}

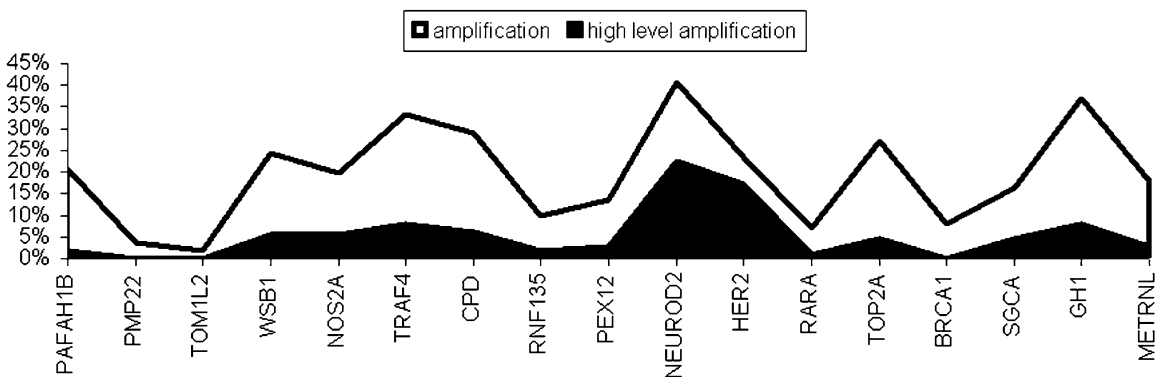

Table 2 Comparison between chromosome 17 centromere copy number by CEP17 CISH and WSB1 MLPA in 104 invasive breast cancer patients (concordance 92.3\%)

\begin{tabular}{llc}
\hline & WSB1 MLPA & \\
\cline { 2 - 3 } & Normal & Increased \\
\hline CEP17 CISH & & \\
$\leq 2$ copies & 82 & 2 \\
$>2$ copies & 6 & 14 \\
\hline
\end{tabular}

centromere status by CEP17 (1-2 copies/nucleus) and MLPA (ratio 1.05). Only two patients (1.8\%) showed amplification of all the tested genes on the $17 \mathrm{q}$ arm, pointing to a gain of whole $17 \mathrm{q}$. These patients had increased centromere copy numbers by CEP17 (3-5 copies/ nucleus and small clusters, respectively) and MLPA (ratio $1.86,1.94$, respectively).

\section{Centromere status and HER2 amplification}

Table 3 shows the association between centromere status by CISH and MLPA, and HER2 amplification. Eight of the 16 patients (50\%) showing amplification of WSB1 and 12/ 20 patients $(60 \%)$ showing amplification of CEP17 were also HER2 amplified. About 55\% (12/22) and 36\% (8/22) of HER2 amplified patients showed CEP17- and WSB1based "polysomy 17," respectively. Only one patient presented with a loss of WSB1 and CEP17, but this was not a
Table 3 The association between centromere status by CEP17 CISH or WSB1 MLPA on the one hand and HER2 amplification by MLPA on the other

\begin{tabular}{llllll}
\hline & \multicolumn{2}{l}{ CEP17 CISH } & & \multicolumn{2}{l}{ WSB1 MLPA } \\
& $\leq 2$ copies & $>2$ copies & & Normal & Increased \\
\hline HER2 status & & 8 & 74 & 8 \\
Normal & 74 & 12 & & 14 & 8 \\
Amplified & 10 & &
\end{tabular}

true monosomy 17 since several other chromosome 17 genes had normal copy numbers.

Association between amplified regions

Most genes were never found amplified or lost alone. Amplification of HER2 (26/111 patients) was often associated with amplification of NEUROD2 (23/26, 88\%), GH1 (17/26, 65\%), TRAF4 (15/26, 58\%), TOP2A $(13 / 26,50 \%)$ and WSB1 (12/26, 46\%). Amplification of WSB1 (18 of 111 patients) was most frequently associated with amplification of TRAF4 (81\%), CPD (74\%), GH1 (70\%), NEUROD2 (67\%), NOS2A (63\%) and TOP2A (59\%).

Cluster analysis (Fig. 2) showed that only HER2 and NEUROD2 were frequently clustered together, which is consistent with other studies [23]. WSB1, NOS2A and TRAF4 seemed to form a second small gene cluster independent of the HER2/NEUROD2 cluster. 
Fig. 2 Hierarchical cluster analysis of 111 invasive breast cancer patients analyzed for copy number status of 17 chromosome 17 genes by MLPA

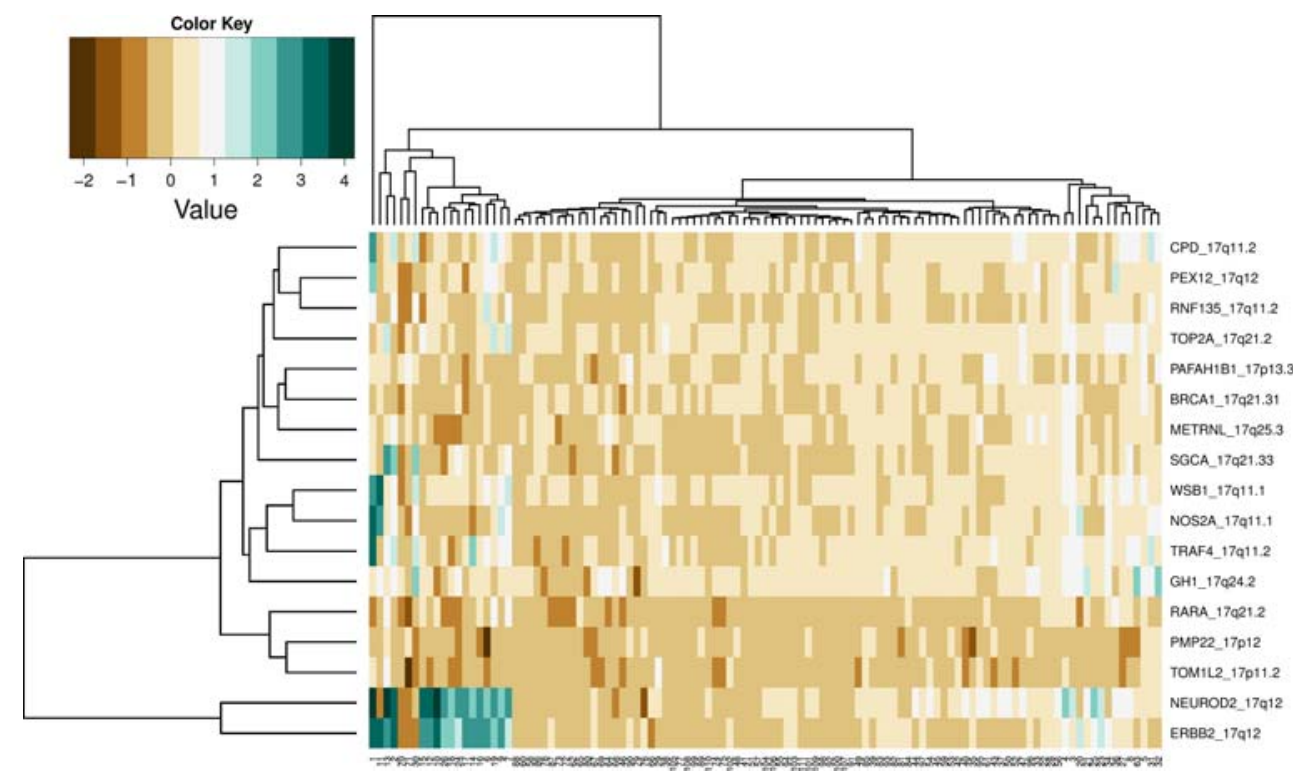

\section{Discussion}

This study aimed to investigate the frequency of polysomy 17 and its association with HER2 gene amplification in invasive breast cancer patients by CEP17 CISH and MLPA analysis. Also, we determined copy number aberrations of a set of genes along chromosome 17 .

Chromosome 17 appears to undergo selective pressure to gain or lose specific regions as exemplified by the frequency plot of chromosome 17 shown in Fig. 1. A number of the events on chromosome 17 are bordered by sharp transitions, and these breakpoints tend to cluster in small intervals $(0.2-2 \mathrm{Mb},[13])$ that could represent fragile sites leading to the occurrence of copy number changes. Consistent with CGH studies, we found complex combinations of gains and losses on chromosome 17q and mainly losses on $17 p[12,13]$. Some studies [12] showed that a fraction of the complex amplification patterns ("firestorms") on 17q (5-10\%) did not include amplification of HER2, giving weight to the notion that other loci in the region may contribute to oncogenesis. In our study, cluster analysis of the analyzed chromosome 17 genes identified especially HER2 and NEUROD2 to be clustered together, which is consistent with other studies [23]. This implies that the HER2/NEUROD2 cluster is the most important amplicon on chromosome 17, which is also confirmed by the high number of high level amplifications in this amplicon when compared to the other chromosome 17 amplicons.

This study identified three other regions of amplification: 17q11.1-11.2 with the WSB1, NOS2A, TRAF4 and CPD genes; 17q21.2 with TOP2A; and 17q21.33-q25.3 with SGCA, GH1 and METRNL. These amplified regions are consistent with CGH studies [12, 13, 15], although the last region can probably be subdivided into more regions if the gene probe density along 17qter is increased in the MLPA mix. Important to notice is the location of HER2 and TOP2A in different amplicons.

In the present study, none of the 111 analyzed patients showed a true polysomy 17 as reflected by simultaneous copy number increase of most genes and no losses along chromosome 17 , although $16 \%$ of patients did show a higher WSB1 copy number and 19\% of patients showed an increased CEP17 copy number. In our study, there was no perfect correlation between CEP17 and WSB1 (92\%) indicating that WSB1 may not completely replace CEP17 for centromere analysis. But more importantly, our data imply that nor WSB1, nor CEP17, can be used to determine true polysomy of chromosome 17 , since amplification of the centromere region by either techniques was not associated with amplification of other chromosome 17 loci. We believe that true polysomy 17 is extremely uncommon in breast cancer. There were no patients showing amplification of whole $17 \mathrm{p}$ and only $2 / 111$ (1.8\%) patients showed possible whole $17 \mathrm{q}$ amplification.

In our study, 50-60\% of the patients showing WSB1- or CEP17-based polysomy 17 were HER2 amplified and up to $55 \%$ of HER2-amplified patients were polysomic based on WSB1 MLPA or CEP17 CISH analysis. The group of Hoffman et al. [24] showed that two "polysomic" patients that were FISH negative (but $\mathrm{IHC} 3+$ ) responded to trastuzumab indicating that FISH analysis can lead to falsenegative results mainly based on CEP17 amplification. The HER2/CEP17 ratio may thus not be the best way to evaluate the HER2 status in all cases and the absolute HER2 gene copy number (whether increased through amplification or polysomy) may be the more important determinant for trastuzumab response for some patients. Except for this 
study, it is presently unknown whether patients with amplicons spanning HER2 and CEP17 will respond to trastuzumab. Vanden Bempt et al. [6] showed that tumors displaying CEP17 amplification in the absence of HER2 amplification resemble more HER2-negative than HER2positive tumors. These findings highlight the need for clinical trials to investigate whether patients with CEP17 amplification benefit from HER2-targeted therapy. At present, there is no clinical indication to determine CEP17 status on itself, although Bartlett et al. [9] did suggest a possible association between CEP17 amplification and response to anthracyclins.

In this study, we used MLPA as a technique to determine copy number changes of a set of genes along chromosome 17. In previous studies, we have already shown a good correlation between MLPA and FISH/CISH for HER2 and a good sensitivity and specificity of MLPA based on CISH as gold standard [18, 25]. Although interpretation of MLPA results is easier than FISH, one major disadvantage of MLPA is that it is a non-morphological technique that requires proper control of input material.

Since we randomly selected the breast tumor samples in this study, only 20/111 of the included patients were HER2 positive. Our results are consistent, however, with recent data from two studies using array CGH on breast tumors, which also suggest that polysomy of chromosome 17 is a rare event in breast cancer [26, 27]. Although extensive prognostic testing should be performed before drawing definite conclusions on the value of CEP17 in HER2 scoring, evidence from our study and other studies is mounting and at least raise questions concerning the proper selection of patients for trastuzumab/lapatinib therapy based on HER2 scoring with CEP17 correction.

In summary, this extensive analysis of amplicons along chromosome 17 shows that true polysomy of chromosome 17, either of the whole chromosome, or the short or the long arm, is likely very rare. Chromosome 17 usually shows a complex pattern of gains and losses, rather unrelated to the copy number status of the centromere and as a consequence, at least in some cases, correction with CEP17 probes may provide misleading HER2 gene status assessment results. Determining what CEP17 amplification means in terms of response to trastuzumab and anthracyclin treatments remains to be further studied. A more comprehensive analysis of amplicons along chromosome 17 rather than just HER2/CEP17 FISH or CISH may be indicated in IHC positive or doubtful breast cancers.

Conflict of interest statement There are no conflicts of interest to be declared.

\section{References}

1. Akiyama T, Sudo C, Ogawara H, Toyoshima K, Yamamoto T (1986) The product of the human c-erbB-2 gene: a 185-kilodalton glycoprotein with tyrosine kinase activity. Science 232:16441646

2. Popescu NC, King CR, Kraus MH (1989) Localization of the human erbB-2 gene on normal and rearranged chromosomes 17 to bands q12-21.32. Genomics 4:362-366

3. Joensuu H, Kellokumpu-Lehtinen PL, Bono P, Alanko T, Kataja V, Asola R, Utriainen T, Kokko R, Hemminki A, Tarkkanen M, Turpeenniemi-Hujanen T, Jyrkkio S, Flander M, Helle L, Ingalsuo S, Johansson K, Jaaskelainen AS, Pajunen M, Rauhala M, Kaleva-Kerola J, Salminen T, Leinonen M, Elomaa I, Isola J (2006) Adjuvant docetaxel or vinorelbine with or without trastuzumab for breast cancer. N Engl J Med 354:809-820

4. Slamon DJ, Leyland-Jones B, Shak S, Fuchs H, Paton V, Bajamonde A, Fleming T, Eiermann W, Wolter J, Pegram M, Baselga J, Norton L (2001) Use of chemotherapy plus a monoclonal antibody against HER2 for metastatic breast cancer that overexpresses HER2. N Engl J Med 344:783-792

5. Hudis CA (2007) Trastuzumab, mechanism of action and use in clinical practice. N Engl J Med 357:39-51

6. Vanden Bempt I, Van LP, Drijkoningen M, Neven P, Smeets A, Christiaens MR, Paridaens R, De Wolf-Peeters C (2008) Polysomy 17 in breast cancer: clinicopathologic significance and impact on HER-2 testing. J Clin Oncol 26:4869-4874. doi: 10.1200/JCO.2007.13.4296

7. Downs-Kelly E, Yoder BJ, Stoler M, Tubbs RR, Skacel M, Grogan T, Roche P, Hicks DG (2005) The influence of polysomy 17 on HER2 gene and protein expression in adenocarcinoma of the breast: a fluorescent in situ hybridization, immunohistochemical, and isotopic mRNA in situ hybridization study. Am J Surg Pathol 29:1221-1227

8. Hyun CL, Lee HE, Kim KS, Kim SW, Kim JH, Choe G, Park SY (2008) The effect of chromosome 17 polysomy on HER-2/neu status in breast cancer. J Clin Pathol 61:317-321. doi:10.1136/ jcp.2007.050336

9. Bartlett JM, Desmedt C, Munro A, O’Malley FP, Larsimont D, Di Leo A, Cameron DA, Isola J, Shepherd L, Twelves CJ, Pritchard KI (2009) Chromosome 17 polysomy: a unifying hypothesis underlying benefit from adjuvant anthracyclines? Cancer Res 69(Suppl. 2): 11

10. Courjal F, Theillet C (1997) Comparative genomic hybridization analysis of breast tumors with predetermined profiles of DNA amplification. Cancer Res 57:4368-4377

11. Forozan F, Mahlamaki EH, Monni O, Chen Y, Veldman R, Jiang Y, Gooden GC, Ethier SP, Kallioniemi A, Kallioniemi OP (2000) Comparative genomic hybridization analysis of 38 breast cancer cell lines: a basis for interpreting complementary DNA microarray data. Cancer Res 60:4519-4525

12. Hicks J, Krasnitz A, Lakshmi B, Navin NE, Riggs M, Leibu E, Esposito D, Alexander J, Troge J, Grubor V, Yoon S, Wigler M, Ye K, Borresen-Dale AL, Naume B, Schlicting E, Norton L, Hagerstrom T, Skoog L, Auer G, Maner S, Lundin P, Zetterberg A (2006) Novel patterns of genome rearrangement and their association with survival in breast cancer. Genome Res 16:14651479. doi:10.1101/gr.5460106

13. Orsetti B, Nugoli M, Cervera N, Lasorsa L, Chuchana P, Ursule L, Nguyen C, Redon R, du MS, Rodriguez C, Theillet C (2004) Genomic and expression profiling of chromosome 17 in breast cancer reveals complex patterns of alterations and novel candidate genes. Cancer Res 64:6453-6460 
14. Andre F, Job B, Dessen P, Tordai A, Michiels S, Liedtke C, Richon C, Yan K, Wang B, Vassal G, Delaloge S, Hortobagyi GN, Symmans WF, Lazar V, Pusztai L (2009) Molecular characterization of breast cancer with high-resolution oligonucleotide comparative genomic hybridization array. Clin Cancer Res 15:441-451. doi:10.1158/1078-0432.CCR-08-1791

15. Pollack JR, Sorlie T, Perou CM, Rees CA, Jeffrey SS, Lonning PE, Tibshirani R, Botstein D, Borresen-Dale AL, Brown PO (2002) Microarray analysis reveals a major direct role of DNA copy number alteration in the transcriptional program of human breast tumors. Proc Natl Acad Sci USA 99:12963-12968. doi: 10.1073/pnas.162471999

16. Arriola E, Marchio C, Tan DS, Drury SC, Lambros MB, Natrajan R, Rodriguez-Pinilla SM, Mackay A, Tamber N, Fenwick K, Jones C, Dowsett M, Ashworth A, Reis-Filho JS (2008) Genomic analysis of the HER2/TOP2A amplicon in breast cancer and breast cancer cell lines. Lab Invest 88:491-503. doi:10.1038/ labinvest.2008.19

17. Schouten JP, McElgunn CJ, Waaijer R, Zwijnenburg D, Diepvens F, Pals G (2002) Relative quantification of 40 nucleic acid sequences by multiplex ligation-dependent probe amplification. Nucleic Acids Res 30:e57

18. Moelans CB, de Weger RA, van Blokland MT, Ezendam C, Elshof S, Tilanus MG, van Diest PJ (2009) HER-2/neu amplification testing in breast cancer by multiplex ligation-dependent probe amplification in comparison with immunohistochemistry and in situ hybridization. Cell Oncol 31:1-10

19. Slamon D, Eiermann W, Robert N (2005) Phase III randomized trial comparing doxorubicin and cyclophosphamide followed by docetaxel (ACT) with doxorubicin and cyclophosphamide followed by docetaxel and trastuzumab (ACTH) with docetaxel, carboplatin and trastuzumab $(\mathrm{TCH})$ in Her2-positive early breast cancer patients: BCIRG006 study.94:S5, 2005 (suppl. 1; abstr 1)

20. Coon JS, Marcus E, Gupta-Burt S, Seelig S, Jacobson K, Chen S, Renta V, Fronda G, Preisler HD (2002) Amplification and overexpression of topoisomerase IIalpha predict response to anthracycline-based therapy in locally advanced breast cancer. Clin Cancer Res 8:1061-1067

21. van Diest PJ (2002) No consent should be needed for using leftover body material for scientific purposes. BMJ 325:648-651

22. Coffa J, van de Wiel MA, Diosdado B, Carvalho B, Schouten J, Meijer GA (2008) MLPAnalyzer: data analysis tool for reliable automated normalization of MLPA fragment data. Cell Oncol 30:323-335

23. Kauraniemi P, Kuukasjarvi T, Sauter G, Kallioniemi A (2003) Amplification of a 280-kilobase core region at the ERBB2 locus leads to activation of two hypothetical proteins in breast cancer. Am J Pathol 163:1979-1984

24. Hofmann M, Stoss O, Gaiser T, Kneitz H, Heinmoller P, Gutjahr T, Kaufmann M, Henkel T, Ruschoff J (2008) Central HER2 IHC and FISH analysis in a trastuzumab (Herceptin) phase II monotherapy study: assessment of test sensitivity and impact of chromosome 17 polysomy. J Clin Pathol 61:89-94. doi:10.1136/ jcp.2006.043562

25. Moelans CB, de Weger RA, Ezendam C, van Diest PJ (2009) HER-2/neu amplification testing in breast cancer by multiplex ligation-dependent probe amplification: influence of manual- and laser microdissection. BMC Cancer 9:4. doi:10.1186/14712407-9-4

26. Yeh IT, Martin MA, Robetorye RS, Bolla AR, McCaskill C, Shah RK, Gorre ME, Mohammed MS, Gunn SR (2009) Clinical validation of an array CGH test for HER2 status in breast cancer reveals that polysomy 17 is a rare event. Mod Pathol. doi: 10.1038/modpathol.2009.78

27. Marchio C, Lambros MB, Gugliotta P, Di Cantogno LV, Botta C, Pasini B, Tan DS, Mackay A, Fenwick K, Tamber N, Bussolati G, Ashworth A, Reis-Filho JS, Sapino A (2009) Does chromosome 17 centromere copy number predict polysomy in breast cancer? A fluorescence in situ hybridization and microarraybased CGH analysis. J Pathol 219:16-24. doi:10.1002/path.2574 